doi: http://dx.doi.org/10.22265/acnef.4.2.287

\title{
Comportamiento diferencial del paciente diabético en relación con el no diabético en hemodiálisis, en el hospital provincial de Camagüey, Cuba
}

\section{Differential behavior of diabetic patients compared to non-diabetic on hemodialysis, at the provincial hospital in Camagüey, Cuba}

\author{
Milene Benítez Méndez¹, Dashiell Millet Torres², Leonardo Curbelo Rodríguez ${ }^{3}$, Francisco Prieto García ${ }^{4}$ \\ ${ }^{1}$ Especialista de Primer Grado en Nefrología, Hospital Universitario Manuel Ascunce Domenech, Camagüey, Cuba \\ ${ }^{2}$ Especialista de Primer grado en Nefrología, Hospital Municipal de Nuevitas, Camagüey, Cuba, \\ Universidad de Ciencias Médicas Carlos J. Finlay, Hospital Universitario Manuel Ascunce Domenech, Camagüey, Cuba \\ ${ }^{3}$ Especialista de Segundo Grado en Nefrología, Máster en Urgencias Médicas, Profesor Instructor. Hospital Universitario Manuel Ascunce Domenech, Camagüey, Cuba \\ ${ }^{4}$ Profesor-Investigador Titular, Nivel C, Doctor en Química, Área Académica de Ciencias Ambientales, Toxicología, Universidad Autónoma del Estado de Hidalgo, Pachuca,
} México

\begin{abstract}
Resumen
Introducción: hoy, la diabetes mellitus (DM) es considerada una verdadera epidemia. Quienes la padecen presentan alto riesgo de desarrollar nefropatía diabética, entre el 20 y $50 \%$ de ellos tiene riesgo de sufrir insuficiencia renal crónica terminal y necesitar de diálisis o trasplante de riñones. Objetivos: evaluar el comportamiento diferencial del paciente diabético en relación con el no diabético en hemodiálisis, del Hospital Universitario Manuel Ascunce Domenech de Camagüey, durante el período de agosto de 2009 hasta enero de 2014.

Metodología: estudio descriptivo, de cohorte de 174 pacientes en hemodiálisis (estadios 3b, 4 y 5). La muestra fue de noventa pacientes clasificados entre diabéticos y no diabéticos, de acuerdo con criterios de inclusión, exclusión y salida. La fuente primaria de información consistió en una encuesta confeccionada según criterios de objetivos trazados. La fuente secundaria estuvo conformada por historias clínicas y pancartas de los pacientes en hemodiálisis. Las variables estudiadas fueron: edad, sexo, comorbilidad, tiempo en hemodiálisis, estado nutricional, tipo y número de intentos de acceso vascular, complicaciones asociadas a hemodiálisis, así como las principales causas que invalidan a dichos pacientes para el trasplante renal.

Resultados: predominaron pacientes mayores de 60 años, masculinos, con menos de un año en hemodiálisis. Las comorbilidades mayormente encontradas fueron: hipertensión arterial, cardiopatía isquémica y hepatopatías, en ese orden. En diabéticos, prevalecieron quienes presentaron infrapeso o sobrepeso ligero.

Conclusiones: las comorbilidades fueron con más frecuencia: hipertensión arterial, cardiopatía isquémica y hepatopatías, en ese orden y en ambos grupos. Se encontraron en mayor número los catéteres doble-luz, así como más intentos de acceso vascular en población diabética. Las complicaciones que más se presentaron en hemodiálisis fueron: hipotensión, hipoglucemia e infecciones. Principalmente, el trasplante renal fue contraindicado por enfermedad cardiovascular y las edades extremas.
\end{abstract}

Palabras clave: hemodiálisis, diabetes mellitus, hipertensión arterial, hepatopatías, enfermedad cardiovascular.

doi: http://dx.doi.org/10.22265/acnef.4.2.287

\begin{abstract}
Introduction: Currently, Diabetes Mellitus (DM) is considered an epidemic. Patients with this disease are at high risk of developing diabetic nephropathy. Previous studies have reported that between $20 \%$ and $50 \%$ of diagnosed patients are at risk of developing chronic renal failure which requires hemodialysis treatment or kidney transplant.

Objectives: The aim of this paper is to evaluate the differential behavior of diabetic patients compared to non-diabetic patients on hemodialysis at University Hospital Manuel Ascunce Domenech in Camagüey, from August 2009 to January 2014.

Methodology: The descriptive study of cohort of 174 hemodialysis patients (stage 3b, 4 and 5) was performed. A sample of 90 patients, including diabetics and non-diabetics, was studied, considering inclusion, exclusion, and exit criteria. The survey was the primary source of information and clinical records of patients on hemodialysis were the secondary source. Age, sex, comorbidity, time on hemodialysis, nutritional status, type of vascular access, number of attempts at attaining vascular access, complications resulting from hemodialysis, and factors impeding the realization of a kidney transplant in diabetic patients, were the variables studied.

Results: This study indicates that male patients, older than 60 years and with hemodialysis periods less than one year, were predominant. The main comorbidities found were arterial hypertension, ischemic heart disease and liver disease. In diabetic patients, underweight and overweight prevailed.

Conclusions: This research has shown that diabetic patients showed the highest number of dual-lumen catheters and more attempts at attaining vascular access. The main complications that resulted from hemodialysis were hypotension, hypoglycemia and infections. Furthermore, it was found that cardiovascular diseases and advanced age were the factors that prevented the realization of a kidney transplant in diabetic patients.
\end{abstract}

Key words: Hemodialysis, diabetes mellitus, hypertension, liver disease, cardiovascular disease.

doi: http://dx.doi.org/10.22265/acnef.4.2.287

\section{(c) $(9)$}

Referenciar este artículo: Benítez Méndez M, Millet Torres D, Curbelo Rodríguez L, Prieto García F. Comportamiento diferencial del paciente diabético en relación con el no diabético en hemodiálisis, en el hospital provincial de Camagüey, Cuba Rev. Colomb. Nefrol. 2017;4(2): 168-187 doi: http://dx.doi.org/10.22265/acnef.4.2.287 Correspondencia: *Francisco Prieto García, e-mail: prietog@uaeh.edu.mx

Recibido: 30-06-17 - Aceptado: 13-08-17 • Publicado en línea: 14-08-17 


\section{Introducción}

$\mathrm{L}$

a diabetes mellitus (DM) es actualmente una verdadera epidemia. Quienes la padecen presentan un alto riesgo de desarrollar nefropatía diabética y entre el 20 y el 50 $\%$ de ellos tiene riesgo de sufrir insuficiencia renal crónica terminal (IRCT) y necesitar de diálisis o trasplante de riñones ${ }^{1-3}$. Se sabe que cerca del $40 \%$ de los casos nuevos de IRCT se debe a esta patología. El número de pacientes diabéticos que inician diálisis está aumentando en todo el mundo y, en la provincia de Camagüey, no es una distinto, sino que constituye un verdadero problema de salud. Estos pacientes presentan diferencias significativas con respecto al resto de pacientes en diálisis, en cuanto a sus características demográficas, complicaciones, comorbilidades y particularidades en su tratamiento. Necesitan, además, un manejo especial en la mayoría de las áreas de la hemodiálisis, como son las pautas de diálisis, el acceso vascular (AV) o el control de la diabetes. Asimismo de la anemia, la vasculopatía y la retinopatía, que suelen tener asociadas estos pacientes $^{4}$.

La insuficiencia renal crónica terminal (IRCT), secundaria a nefropatía diabética (ND), es la causa más común de ingreso en programas de terapia renal sustitutiva (TSR). El incremento de la afectación por DM está relacionado con una mayor exposición a factores de riesgo (hábitos dietéticos inadecuados, sedentarismo, obesidad, entre otros) y con el aumento de la expectativa de vida. Así, un mayor número de personas alcanza las edades en que esta enfermedad es más frecuente ${ }^{5}$.

La disminución de la mortalidad infantil, de enfermedades transmisibles y de la tasa de mortalidad general ha producido un aumento de la expectativa de vida de la población cubana (actualmente 77 años). Se ha originado un envejecimiento de la población, que producirá un continuo aumento de prevalencia de DM durante los próximos 30 años, a menos que se desarrolle una mayor eficacia en el control de los factores de riesgo más importantes (sedentarismo, obesidad) ${ }^{6}$.

Al despuntar este siglo, se desencadenó la llamada "epidemia global de diabetes", fenómeno relacionado particularmente con la DM tipo 2 y que está teniendo lugar tanto en países desarrollados como en países en vía de desarrollo ${ }^{6}$. La ND raramente aparece antes de los 10 años de evolución de la DM. Sin embargo, en algunos casos, puede presentarse antes de ese tiempo e, incluso, suceder antes de la DM. Ocasionalmente, puede detectarse microalbuminuria en pacientes con más de 12 años de evolución de la enfermedad ${ }^{7}$. En consecuencia, se estima que la prevalencia mundial de DM tipo 2 aumentará, de los 171 millones que había en el año 2000, a una cifra de, al menos, 366 millones para el año 2030. Por tanto, un mayor número de personas llegará a padecer IRCT y requerir terapia renal sustitutiva $a^{6,8}$. Las así llamadas "complicaciones microvasculares" (retinopatía y nefropatía) son responsables directas de la morbilidad más frecuentemente asociada a la DM. En España, el porcentaje de pacientes diabéticos con IRCT supone un $36 \%$ del total y la causa más importante de insuficiencia renal terminal $(26 \%)$ es la ND ${ }^{9}$. En Alemania y en los EE. UU., más del $40 \%$ de las personas con IRCT tiene diabetes. A nivel global, el número de pacientes con esta patología y que, además, necesitaban diálisis fue de 1.000 .000 en 2005, de los cuales 260.000 residían en los EE. UU. Se calcula que esta población crecerá a un ritmo anual del $8 \%$.

A nivel mundial, la IRCT es una de las patologías de mayor impacto, que se ha ubicado en los últimos 7 años dentro de las primeras causas de morbimortalidad. En Venezuela, estas cifras se corresponden a las registradas en países como Chile, Argentina, Uruguay y Colombia10, donde la DM ocupa la principal causa, entre el 30 y $40 \%$. En un estudio realizado en diez países de Latinoamérica, incluida Venezuela, se determinó que la ND fue la causa más frecuente de IRCT con un $24,6 \% 11,12$. Según este estudio, en Venezuela hay 9.408 pacientes en diálisis y 2.122 de ellos en hemodiálisis $(79 \%){ }^{10,13}$. 
En Cuba, también se ha observado un incremento progresivo de afectados con IRCT aceptados en los programas de diálisis y trasplante renal (TR). Han alcanzado el $10 \%$ de la totalidad en los últimos años, con propensión al aumento ${ }^{5,13,14}$. En el 2003, existían aproximadamente 14.052 personas con $\mathrm{DM}$ en la provincia de Santiago de Cuba. De ellas, 1.054 fueron diagnosticadas como tales ese mismo año y, en el 2004, aumentó la cifra a 1.276 nuevos pacientes ${ }^{15}$. Según estadísticas del Servicio de Nefrología de Camagüey, del total de pacientes incluidos en el Programa de crónicos en el año 2012, el $11 \%$ fue constituido por pacientes diabéticos, quienes aumentan el índice de defunciones en el año.

$\mathrm{Si}$, además de lo anterior, consideramos que la esperanza de vida del diabético es, en promedio, ocho años menor que la de personas no diabéticas en edades de 55 a 64 años y cuatro años menor en los de 65 a 747 , podemos ratificar que esta afección constituye, cada vez más, un importante problema de salud ${ }^{7,8}$. Por causa del envejecimiento de la población mundial, hacia el 2025, padecerán diabetes 146 millones de personas entre los 40 y los 59 años y 147 millones de personas con 60 años o más ${ }^{11}$. Globalmente, la diabetes demanda tantas vidas al año como el VIH/SIDA ${ }^{16-18}$.

En Cuba, la tasa continúa más alta en mujeres que en hombres $(41,5 \%$ frente a $25 \%) \mathrm{y}$, desde hace varios años, se ubica entre las primeras diez causas de muerte ${ }^{19,20}$. En el año 2010, ocasionó 2.638 defunciones, para una tasa de 23,5 x 100.000 habs $^{21}$. Se observa, además, un crecimiento sostenido de los enfermos prevalentes en métodos dialíticos, con una tasa de 119 por millón poblacional (pmp) en 2008, de 134 pmp en 2009 y de 149 pmp en 2010, lo que significa un incremento anual del 10,3\%, 11,2 $\%$ y $11,1 \%$ respectivamente ${ }^{22-24}$.

La Organización Panamericana de la Salud (OPS) calcula que el costo de la diabetes y la IRCT en la región es de, al menos, 65 mil millones de dólares al año, resultado de muerte prematura, ausentismo laboral, discapacidad, medicamentos, hospitalizaciones y consultas médicas ${ }^{25-27}$.

La probabilidad de desarrollar una IRCT varía considerablemente en función de características raciales y étnicas en los pacientes con DM. Se ha estimado que, en los pacientes con Diabetes tipo 2 de raza caucásica, se encuentra en el rango del 15 al $20 \%$. Sin embargo, la prevalencia de diabetes tipo 2 es de 10 a 15 veces mayor que la de la DM tipo 1. El resultado es que entre el 60 y el $90 \%$ de los pacientes diabéticos que inician programas de diálisistrasplante presentan DM 2. El envejecimiento relativo de la población, el incremento de DM 2 y la mejoría en la supervivencia de estos pacientes conduce a que un importante número de pacientes desarrolle IRC, secundaria a ND ${ }^{28}$.

La historia natural de la ND se entiende como un camino progresivo, desde las alteraciones funcionales renales, hasta la insuficiencia renal terminal. Atraviesa estadios intermedios, marcados por la aparición de microalbuminuria y proteinuria $^{29}$. Puede progresar rápidamente, tras períodos relativamente largos de estabilidad, o finalizar con la muerte precoz del paciente, por causa cardiovascular generalmente. El curso clínico de la nefropatía, en los pacientes con DM tipo 2, puede presentar diferencias significativas comparado con el de los pacientes con DM tipo 1, en las fases previas al desarrollo de la proteinuria establecida. Debido a una edad más avanzada, así como a la presencia de arterioesclerosis, los pacientes con DM tipo 2 pueden presentar otras enfermedades renales, sobreañadidas, con más frecuencia que los pacientes con DM tipo 1, incluso, a la nefropatía diabética. La suma de los daños multisistémicos de la DM y la insuficiencia renal crónica hacen muy difícil el tratamiento a estos enfermos ${ }^{30}$.

Las primeras alteraciones encontradas en los pacientes con ND son microalbuminuria e hiperfiltración glomerular, lo que implica un engrosamiento de la membrana basal glomerular, hipertrofia tubular y expansión de la matriz mesangial. Este es, posiblemente, un mecanismo 
compensatorio para prevenir la pérdida urinaria de agua y electrolitos, sobrecarga que genera daño glomerular ${ }^{33} \mathrm{y}$, a su vez, disminuye el área de filtración glomerular (FG), con pérdida progresiva de la función renal. Por último, se observa glomeruloesclerosis y enfermedad renal terminal ${ }^{31,32}$.

El término ND se utiliza, exclusivamente, para señalar las lesiones renales originadas por afección microangiopática o de los pequeños vasos ${ }^{33}$. El cuadro clínico se caracteriza por proteinuria persistente, hipertensión arterial y deterioro progresivo de la función renal ${ }^{34,35}$. La hiperglucemia y la hipertensión arterial, combinadas, producen importantes consecuencias clínicas ${ }^{4,12}$.

La IRCT se define como la entidad final a la que pueden arribar las enfermedades renales crónicas (ERC), primarias o secundarias. Su característica fundamental es el deterioro progresivo e irreversible de la función renal global, con un nivel de FG menor de $15 \mathrm{~mL} /$ minutos/1,73 $\mathrm{m}^{2}$ de superficie corporal ${ }^{36}$.

Debido al fracaso de la función renal, los pacientes con ND son incluidos en el programa de Terapia Renal Sustitutiva (TRS). En todos los pacientes con IRC, la remisión temprana al nefrólogo es importante, pues se asocia a menor mortalidad, mejor nivel de hemoglobina y de albúmina, así como menor estancia hospitalaria al inicio de la diálisis ${ }^{37}$. Para los pacientes diabéticos, este hecho es aún más importante, pues permite una mejor preparación y se pueden aplicar medidas tempranas que eviten la progresión de la insuficiencia renal. Entre estas, podemos citar la intensificación del control glucémico y de la presión arterial, especialmente si existe proteinuria, y la incidencia en los cambios de hábitos de vida poco saludables (peso excesivo, consumo de tabaco, etc.). También se facilita la realización de un AV para un inicio de diálisis cómodo y seguro. El momento óptimo para el inicio de la diálisis está aún por definir. Sin embargo, actualmente, se considera que el mejor momento es cuando no han aparecido manifestaciones urémicas ni signos de malnutrición ${ }^{28}$. Para el caso de los pacientes diabéticos, algunos autores aconsejan que la diálisis se inicie con aclaramientos de creatinina más elevados con respecto a los no diabéticos, en torno a $12-15 \mathrm{~mL} / \mathrm{min}$. Esto es debido a la rápida progresión de la insuficiencia renal en tales pacientes, a las comorbilidades que presentan y al difícil control de la hipertensión arterial cuando el filtrado es tan bajo, con la consecuente aceleración de la retinopatía hipertensiva ${ }^{29}$ y el difícil manejo del sodio y el agua, asociados, frecuentemente, a la aparición de insuficiencia cardíaca ${ }^{40-41}$.

La disfunción del sistema nervioso autónomo y la disfunción diastólica cardíaca del paciente diabético provocan mayor número de hipotensiones en HD. Además, conseguir un buen $\mathrm{AV}$ es difícil debido a la coexistencia, a menudo, de enfermedad vascular. Las hipotensiones tienen un efecto negativo para el paciente diabético y pueden provocar cuadros de angina, isquemia intestinal, cerebral o de miembros inferiores. Este alarmante cuadro se produce por:

- Peor adaptación a la disminución de volumen plasmático de la diálisis, debido a la disfunción autonómica.

- Presencia de anemia.

- Hipoalbuminemia por malnutrición o por un síndrome nefrótico asociado. Este hecho disminuye la presión oncótica, retarda el rellenado vascular y dificulta la recuperación de la hipotensión ${ }^{42}$.

En la provincia, no existen estudios donde se describa el comportamiento del paciente diabético en hemodiálisis y que le sirvan al médico de asistencia como instrumento para actuar sobre la morbimortalidad de estos pacientes y para mejorar, así, su modo de vida. El objetivo central de este trabajo ha sido evaluar el comportamiento diferencial del paciente diabético en relación con el no diabético en hemodiálisis del Hospital Universitario Manuel Ascunce Domenech, de Camagüey, Cuba, en el período comprendido entre agosto de 2009 y enero de 2014. 


\section{Metodología}

Se realizó un estudio descriptivo de cohorte transversal. El universo de estudio estuvo constituido por todos los pacientes con IRCT, a quienes se realizaba hemodiálisis en el Hospital Universitario Manuel Ascunce Domenech' de la Provincia de Camagüey. La muestra estuvo conformada por 174 pacientes en total (estadio 3b, 4 y 5), 90 de los cuales fueron seleccionados a partir de un muestreo aleatorio simple, con el fin de distribuir homogéneamente los pacientes con IRCT tanto por ND, como por otras causas. A su vez, se consideraron aquellos pacientes que cumplieran con los siguientes criterios de inclusión, exclusión y salida:

\section{Criterios de inclusión:}

- Estar de acuerdo con la participación en la investigación, mediante la firma del consentimiento informado.

- Haber cumplido dieciocho años.

\section{Criterios de exclusión:}

- No cumplir con criterios de inclusión.

- No desear participar en el estudio en algún momento de la investigación.

\section{Criterios de salida:}

- Cambiar de centro HD durante el estudio.

- Tener como método sustitutivo renal la diálisis peritoneal, o pasar de hemodiálisis a diálisis peritoneal en algún momento del estudio.

- Recibir TR durante el periodo de estudio.

- Fallecimiento durante el proceso de la investigación.

\section{Obtención de la información}

El dato primario para la investigación se obtuvo a partir de la base de datos del Servicio de Nefrología. La fuente primaria de obtención de la información fue la encuesta, confeccionada según criterios de objetivos trazados. La fuente secundaria estuvo conformada por las historias clínicas y las pancartas de los pacientes en hemodiálisis. En la encuesta se contemplaron las siguientes variables:

- Grupo de edades

- Sexo

- Tiempo en hemodiálisis

- Comorbilidad

- Estado nutricional

- Tipo de acceso vascular

- Intento de accesos vasculares

- Complicaciones en hemodiálisis

- Causas de invalidez del trasplante renal

\section{Procesamiento estadístico}

Se elaboró un fichero de datos con la utilización del paquete de Programa Estadístico SPSS versión 15 , previo al procesamiento de los mismos y la obtención de los resultados. La información obtenida fue procesada en un computador con instalación del sistema Windows XP. Los métodos empleados fueron estadísticas descriptivas y distribución de frecuencias $(p<0,05)$. Los resultados obtenidos se presentaron en tablas y gráficos diseñados al efecto, en las que se resumió la información, con el fin de abordar cada objetivo específico planteado. Posteriormente, se analizó cada resultado obtenido y luego, a través del proceso de síntesis y generalización, se elaboraron conclusiones.

\section{Metódica}

En este apartado se describe la operacionalización de las variables (tabla 1).

\section{Control Semántico}

Acceso Vascular: en hemodiálisis, hay tres modos primarios de acceso a la sangre: el catéter intravenoso, la fístula arteriovenosa interna de Cimino-Brescia (AV) y el injerto sintético. El tipo de acceso está influenciado por factores como el curso previsto del tiempo de la falla renal de un paciente y la condición de su vascularidad. Los pacientes pueden tener múltiples accesos en un tiempo determinado, puesto que, usualmente, un catéter debe ser usado de manera temporal para realizar la diálisis, mientras se está madurando el acceso permanente, la fístula o el injerto arteriovenoso ${ }^{43}$. 
Tabla 1.

Operacionalización de las variables

\begin{tabular}{|c|c|c|c|c|}
\hline \multirow{2}{*}{ Variable } & \multirow{2}{*}{ Tipo de Variable } & \multicolumn{2}{|l|}{ Operacionalización } & \multirow{2}{*}{ Indicador } \\
\hline & & Escala & Descripción & \\
\hline $\begin{array}{l}\text { 1. Grupo de } \\
\text { edades }\end{array}$ & $\begin{array}{l}\text { Cuantitativa } \\
\text { continua }\end{array}$ & $\begin{array}{l}\cdot 18-31 \\
\cdot 32-45 \\
\cdot 46-59 \\
\cdot 60 \text { y más }\end{array}$ & $\begin{array}{l}\text { Se determinará acorde } \\
\text { con la edad referida por } \\
\text { los pacientes o familiares }\end{array}$ & $\begin{array}{c}\text { Distribución de } \\
\text { frecuencias y porcientos. }\end{array}$ \\
\hline 2. Sexo & $\begin{array}{c}\text { Cualitativa nominal } \\
\text { dicotómica }\end{array}$ & $\begin{array}{l}\text { - Femenino } \\
\text { - Masculino }\end{array}$ & $\begin{array}{c}\text { Según sexo biológico de } \\
\text { pertenencia }\end{array}$ & $\begin{array}{c}\text { Distribución de } \\
\text { frecuencias y porcientos }\end{array}$ \\
\hline $\begin{array}{l}\text { 3. Tiempo en } \\
\text { Hemodiálisis }\end{array}$ & $\begin{array}{l}\text { Cuantitativa } \\
\text { continua }\end{array}$ & $\begin{array}{l}\cdot<6 \text { meses } \\
\cdot 6 \text { meses a } 1 \text { año } \\
\cdot 5 \text { de } 10 \text { años } \\
\cdot>10 \text { años }\end{array}$ & $\begin{array}{c}\text { Según el tiempo que } \\
\text { lleve realizándose la } \\
\text { hemodiálisis, referido en } \\
\text { la encuesta. }\end{array}$ & $\begin{array}{l}\text { Distribución de } \\
\text { frecuencias y porcientos }\end{array}$ \\
\hline 4. Comorbilidad & $\begin{array}{l}\text { Cualitativa ordinal } \\
\text { politómica }\end{array}$ & $\begin{array}{l}\text { - Hipertensión arterial } \\
\text { - Cardiopatía isquémica } \\
\text { - Hepatopatías } \\
\text { - Enfermedad vascular periférica } \\
\text { - Enfermedad pulmonar obstructiva crónica } \\
\text { - Enfermedad cerebrovascular }\end{array}$ & $\begin{array}{l}\text { Según datos consignados } \\
\text { en la historia clínica. }\end{array}$ & $\begin{array}{l}\text { Distribución de } \\
\text { frecuencias y porcientos }\end{array}$ \\
\hline $\begin{array}{l}\text { 5. Estado } \\
\text { nutricional }\end{array}$ & $\begin{array}{l}\text { Cuantitativa } \\
\text { continua }\end{array}$ & $\begin{array}{l}\cdot<18 \mathrm{Kg} / \mathrm{m}^{2} \text { Infrapeso } \\
\cdot 18-24,9 \mathrm{Kg} / \mathrm{m}^{2} \text { Normopeso } \\
\text { • } 25-27,9 \mathrm{Kg} / \mathrm{m} 2 \text { Sobrepeso ligero. } \\
\text { • } 28-29,9 \mathrm{Kg} / \mathrm{m} 2 \text { Sobrepeso moderado. } \\
\cdot \text { - } 30-34,9 \mathrm{Kg} / \mathrm{m} 2 . \text { Obesidad Grado I } \\
\cdot 35-39,9 \mathrm{Kg} / \mathrm{m} 2 \text { Obesidad Grado II } \\
\text { - } 40 \text { y más Kg/m2 Obesidad Grado III }\end{array}$ & $\begin{array}{l}\text { Según el peso y la talla, } \\
\text { se determina el IMC, } \\
\text { datos recogidos en la } \\
\text { historia clínica. }\end{array}$ & $\begin{array}{l}\text { Distribución de } \\
\text { frecuencias y porcientos }\end{array}$ \\
\hline $\begin{array}{l}\text { 6. Tipo de acceso } \\
\text { vascular }\end{array}$ & $\begin{array}{l}\text { Cualitativa ordinal } \\
\text { politómica }\end{array}$ & $\begin{array}{l}\text { - Fístula arteriovenosa } \\
\text { - Catéter doble luz } \\
\text { - Injerto protésico. } \\
\text { - Dos a la vez. }\end{array}$ & $\begin{array}{l}\text { Según datos consignados } \\
\text { en la historia clínica. }\end{array}$ & $\begin{array}{l}\text { Distribución de } \\
\text { frecuencias y porcientos }\end{array}$ \\
\hline $\begin{array}{l}\text { 7. Intentos de } \\
\text { Acceso vascular }\end{array}$ & Cuantitativa discreta & $\begin{array}{l}\text { - Menos de } 5 \\
\text { - Entre } 6 \text { y } 10 \\
\text { - Más de } 10\end{array}$ & $\begin{array}{l}\text { Según datos consignados } \\
\text { en la historia clínica. }\end{array}$ & $\begin{array}{l}\text { Distribución de } \\
\text { frecuencias y porcientos }\end{array}$ \\
\hline $\begin{array}{l}\text { 8. Complicaciones } \\
\text { de la Hemodiálisis }\end{array}$ & $\begin{array}{l}\text { Cualitativa ordinal } \\
\text { politómica }\end{array}$ & $\begin{array}{l}\text { - Infecciones } \\
\text { - Hipotensión } \\
\text { - Hipoglucemia } \\
\text { - Arritmias } \\
\text { - Síndrome Coronario Agudo } \\
\text { - Sangramiento } \\
\text { - Parestesias }\end{array}$ & $\begin{array}{l}\text { Según datos consignados } \\
\text { en la historia clínica. }\end{array}$ & $\begin{array}{l}\text { Distribución de } \\
\text { frecuencias y porcientos }\end{array}$ \\
\hline $\begin{array}{l}\text { 9. Principales } \\
\text { Causas de } \\
\text { invalidez para el } \\
\text { trasplante renal }\end{array}$ & $\begin{array}{l}\text { Cualitativa ordinal } \\
\text { politómica }\end{array}$ & $\begin{array}{l}\text { - Enfermedad cardiovascular } \\
\text { - Infección activa } \\
\text { - Enfermedad ulceropéptica activa } \\
\text { - Negativa del paciente } \\
\text { - Enfermedad cerebrovascular reciente } \\
\text { - Edades extremas del paciente } \\
\text { - Enfermedad Neoplásica reciente } \\
\text { - Drogodependencias } \\
\text { - Trastornos Psiquiátricos graves }\end{array}$ & $\begin{array}{l}\text { Según datos consignados } \\
\text { en la historia clínica. }\end{array}$ & $\begin{array}{l}\text { Distribución de } \\
\text { frecuencias y porcientos }\end{array}$ \\
\hline
\end{tabular}


Síndrome de robo arterial: es una complicación poco frecuente del AV requerido en $\mathrm{HD}$, que se produce normalmente dentro de los treinta días siguientes a la cirugía. Desaparece o mejora en pocas semanas. Se manifiesta con frialdad, entumecimiento de los dedos, dolor y palidez en la mano y parestesia. En casos extremos, desemboca en úlceras y gangrena. Se produce por la disminución del flujo arterial de la parte distal de la extremidad, al desviarse parte del riego sanguíneo hacia la fístula arteriovenosa o injerto protésico, ya que estos ofrecen menos resistencia al paso de la sangre. Produce una isquemia en los tejidos ${ }^{44,45}$.

Invalidez para el trasplante renal: son aquellas contraindicaciones, absolutas y relativas, que imposibilitan la realización del TR. Los efectos colaterales asociados a la inmunosupresión conllevan un aumento de riesgo cardiovascular, ya elevado en estos pacientes, de infecciones y de procesos neoplásicos ${ }^{46}$.

\section{Aspectos éticos y procedimiento de campo}

La investigación se realizó conforme a principios éticos para las investigaciones biomédicas en seres humanos. De acuerdo con esto, se solicitó el consentimiento informado de los pacientes; se les explicaron los objetivos de la investigación; se les pidió colaboración, bajo el compromiso de utilizar la información solo en el contexto del estudio, y se les comunicó que estaban en la libertad de negarse, si así lo deseaban.

\section{Resultados y discusión}

La ND es la principal complicación crónica de la DM tipo 2, una enfermedad de alto costo social y económico y la causa principal de ingreso a tratamiento dialítico en todo el mundo. Existe un $21 \%$ de riesgo de desarrollar ND en la DM tipo 2 en los primeros veinte años de evolución de la enfermedad. Cuando se hace evidente clínicamente, el promedio de supervivencia es de siete años para ambos sexos y, si los niveles de creatinina superan la cifra de $176 \mathrm{mmol} / \mathrm{l}^{2}$, de dos años ${ }^{47}$.
Estos pacientes presentan diferencias significativas con el resto de pacientes en diálisis, en cuanto a características demográficas, complicaciones, comorbilidades y objetivos de tratamiento. La totalidad de ellos se encuentran en estadios avanzados de la IRCT, es decir, en estadios $3 \mathrm{~b}$ en adelante. Necesitan un manejo especial en la mayoría de áreas de la HD, como son las pautas de diálisis, el AV o el control de la diabetes, la anemia, la vasculopatía y la retinopatía, enfermedades que suelen tener asociadas estos pacientes ${ }^{47,48}$.

La tabla 2 muestra la distribución de pacientes estudiados según grupo de edades (todos en estadio 3b, 4 y 5). Predominan pacientes con 60 años o más, quienes constituyen el 54,45 \% de los casos y, de estos, el 31,12 \% son diabéticos. Igual tendencia se observó con respecto a la inversión de la pirámide poblacional en España, que ha sido consecuencia de una mejora en la expectativa de vida y se sitúa en 80,2 años, según datos del año 2013. En consecuencia, la incidencia de ERC terminal es mucho mayor en pacientes ancianos que en la población general y, entre aquellos que inician TSR, es tres veces superior a la media en el grupo de mayores de 75 años ${ }^{31}$.

En algunas comunidades autónomas, el número de pacientes mayores de 80 años con ERC terminal se ha multiplicado por diez en los últimos diez años ${ }^{33}$. Es necesario considerar que la disminución del FG, en relación con la edad, no se puede explicar solamente por un proceso involutivo, pues es posible encontrar población con edad avanzada y un FG normal. Lindeman et al. y Rowe et al., en clásicos estudios prospectivos, muestran un descenso progresivo del FG, estimado en $1,09 \mathrm{ml} / \mathrm{min} /$ año. Sin embargo, en un tercio de los individuos de la muestra no se presenta esto; sino que, por el contrario, en un reducido grupo, sí aumenta. Estos datos, además, coinciden con los de otros autores $^{49-51}$. 
Tabla. 2

Característica diferencial del paciente diabético en relación con el no diabético en hemodiálisis. Distribución de los pacientes según grupo de edades y sexos (todos en estadio 3 b y más). Servicio de Nefrología. Hospital Universitario Manuel Ascunce Domenech.2009-2014.

\begin{tabular}{|c|c|c|c|c|c|}
\hline \multirow[t]{2}{*}{ Grupo de Edades } & \multicolumn{2}{|c|}{$\begin{array}{c}\text { IRCT Nefropatía } \\
\text { Diabética }\end{array}$} & \multicolumn{2}{|c|}{$\begin{array}{l}\text { IRCT Causas } \\
\text { No Diabéticas }\end{array}$} & \multirow{2}{*}{$\begin{array}{c}\text { Total } \\
\text { No. }\end{array}$} \\
\hline & No. & $\%$ & No. & $\%$ & \\
\hline 18-31 años & 2 & 2,22 & $\begin{array}{c}1 \\
3,33\end{array}$ & 1,11 & 3 \\
\hline $32-45$ años & 4 & 4,44 & $\begin{array}{c}7 \\
12,22\end{array}$ & 7,78 & 11 \\
\hline 46-59 años & 11 & 12,22 & $\begin{array}{c}16 \\
30,00\end{array}$ & 17,78 & 27 \\
\hline 60 y más & 28 & 31,12 & $\begin{array}{c}21 \\
54,45\end{array}$ & 23,33 & 49 \\
\hline Total & 45 & 50,00 & $\begin{array}{c}45 \\
100,00\end{array}$ & 50,00 & 90 \\
\hline \multirow[t]{2}{*}{ Sexo } & \multicolumn{2}{|c|}{$\begin{array}{c}\text { IRCT Nefropatía } \\
\text { Diabética }\end{array}$} & \multicolumn{2}{|c|}{$\begin{array}{l}\text { IRCT Causas } \\
\text { No Diabéticas }\end{array}$} & Total \\
\hline & No. & $\%$ & No. & $\%$ & No. \\
\hline Masculino & 32 & 35,56 & $\begin{array}{c}25 \\
63,33\end{array}$ & 27,77 & 57 \\
\hline Femenino & 13 & 14,44 & $\begin{array}{c}20 \\
36,67\end{array}$ & 22,23 & 33 \\
\hline Total & 45 & 50,00 & $\begin{array}{c}45 \\
100,00\end{array}$ & 50,00 & 90 \\
\hline
\end{tabular}

Fuente: Base de datos Servicio Nefrología

Ciertamente, el curso clínico de la ERC en pacientes ancianos es poco conocido. Cuando se estratifica el riesgo de mortalidad en población anciana, se objetiva que el riesgo relativo, aunque elevado, es menor que en poblaciones más jóvenes y se observa una atenuación edad, dependiente de la relación entre FG disminuido y mortalidad. Aunque las tasas de mortalidad y ERCT se relacionan, inversamente, con el FG para todas las edades, entre pacientes con FG comparables, los más ancianos tienen un mayor riesgo de mortalidad y un menor riesgo de desarrollar IRCT que los más jóvenes. La relación entre edad, progresión de la enfermedad renal y $\mathrm{FG}$ es compleja. Entre aquellos pacientes con $\mathrm{FG}<45 \mathrm{ml} / \mathrm{min} / 1,73 \mathrm{~m}^{2}$, los que tienen un descenso superior a $3 \mathrm{ml} / \mathrm{min} / 1,73 \mathrm{~m}^{2}$ son los más jóvenes $y$, por el contrario, en aquellos con $\mathrm{FG}$ $>45 \mathrm{ml} / \mathrm{min} / 1,73 \mathrm{~m}^{2}$, el mismo descenso del FG corresponde a las personas de mayor edad ${ }^{52}$. En consecuencia, y admitiendo que la longevidad es el resultado de una mejoría de la salud y que la ERC avanzada depende más de las comorbilidades que de la edad per se, la prevalencia de ERC puede no tener el incremento esperado.

En la tabla 2 se observa también la distribución de los pacientes según el sexo. Pudo verse un predomino del sexo masculino, representado por el $63,33 \%$ de los estudiados. En estudios demográficos realizados en Colombia, se vio, coincidentemente, que el $74,3 \%$ perteneció al sexo masculino y el $25,7 \%$, al femenino ${ }^{51}$.

La figura 1 muestra la relación de los pacientes estudiados según tiempo en HD. Puede verse un mayor número de pacientes recibiendo dicho tratamiento en un período entre seis meses y un 


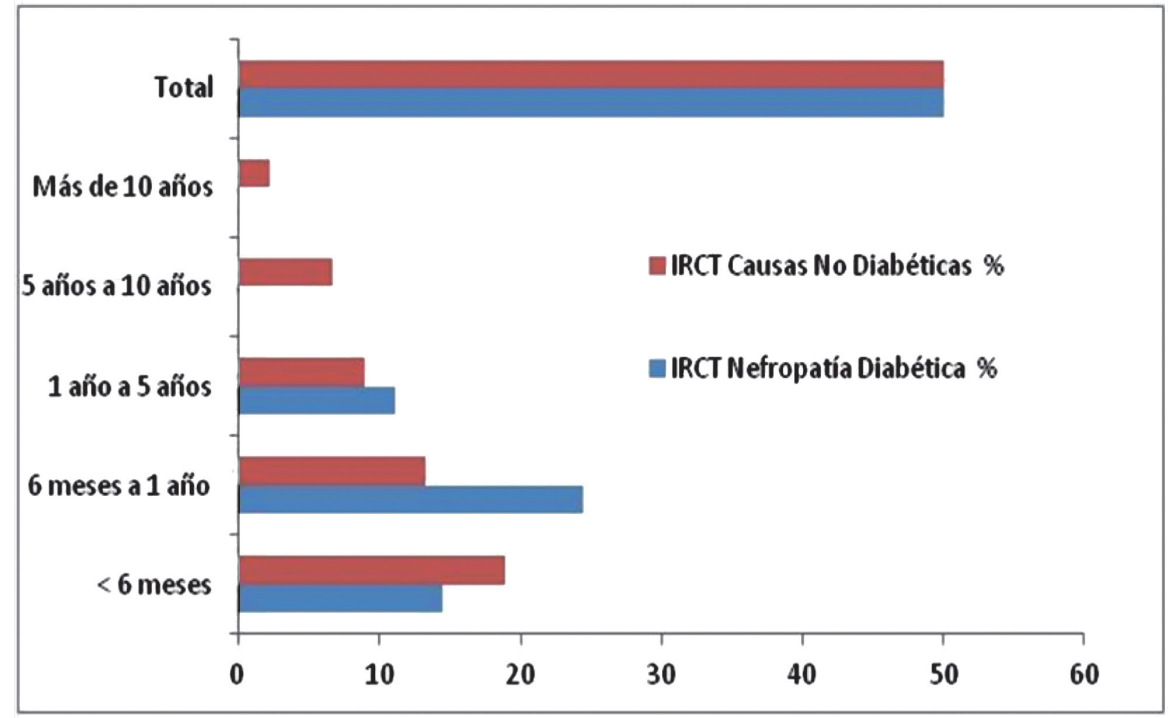

Figura 1. Relación de pacientes estudiados según tiempo en hemodiálisis

año $(37,76 \%)$, con diferencias significativas entre la población no diabética y la diabética. Globalmente, muestra menor supervivencia una vez instaurado el tratamiento dialítico, dada por una mayor comorbilidad en este grupo con aparición de trastornos cardiovasculares, como arritmias y síndrome coronario agudo. Este último, incluso, puede cursar de forma silente ${ }^{52,53}$. También, se manifiestan en mayor medida complicaciones infecciosas, relacionadas o no con el AV, sobre todo cuando se trata de catéter intravascular.

Pese a la mejora sustancial experimentada en todas las formas de tratamiento sustitutivo de la ERC, los pacientes en diálisis continúan sufriendo una significativa morbimortalidad y una calidad de vida reducida ${ }^{54}$. Sus posibilidades de supervivencia son de diez a veinte veces inferiores a las de la población general. En los pacientes con ERC secundaria a DM, la mortalidad anual se incrementa un $40 \%$ más. La enfermedad cardiovascular es la principal causa de muerte en los pacientes en tratamiento con diálisis. Más del $50 \%$ de los pacientes en diálisis tiene tres o más condiciones de comorbilidad, el número de días de hospitalización es superior a quince, por paciente y año, y la calidad de vida referida por ellos es muy inferior a la que requiere la población general. Los pacientes en tratamiento renal sustitutivo, a pesar de los avances en su seguimiento y tratamiento, presentan las peores tasas de supervivencia, morbimortalidad y calidad de vida, debido a su mayor comorbilidad y a que padecen, con mayor frecuencia, insuficiencia cardíaca, cardiopatía isquémica, vasculopatía periférica y hepatopatía crónica. Los pacientes con DM presentan, además, una peor situación funcional y una peor percepción de su salud.

En el caso de los pacientes diabéticos, algunos autores aconsejan que la diálisis se inicie con aclaramientos de creatinina más elevados, entre 12 y $15 \mathrm{~mL} / \mathrm{min}^{55,56}$. Esto es debido a la rápida progresión de la insuficiencia renal en tales pacientes, a las comorbilidades que presentan y al difícil control de la hipertensión arterial cuando el filtrado es tan bajo, con la consecuente aceleración de la retinopatía hipertensiva y el difícil manejo del sodio y el agua, asociado, muy frecuentemente, a la aparición de insuficiencia cardíaca ${ }^{57,58}$.

La figura 2 se refiere a la comorbilidad en los pacientes. Pudo verse un predominio de la hipertensión arterial, la cardiopatía isquémica y las hepatopatía en el caso de la población diabética (88,88 \%, 64,44 \% y 55,55\%, respectivamente). 


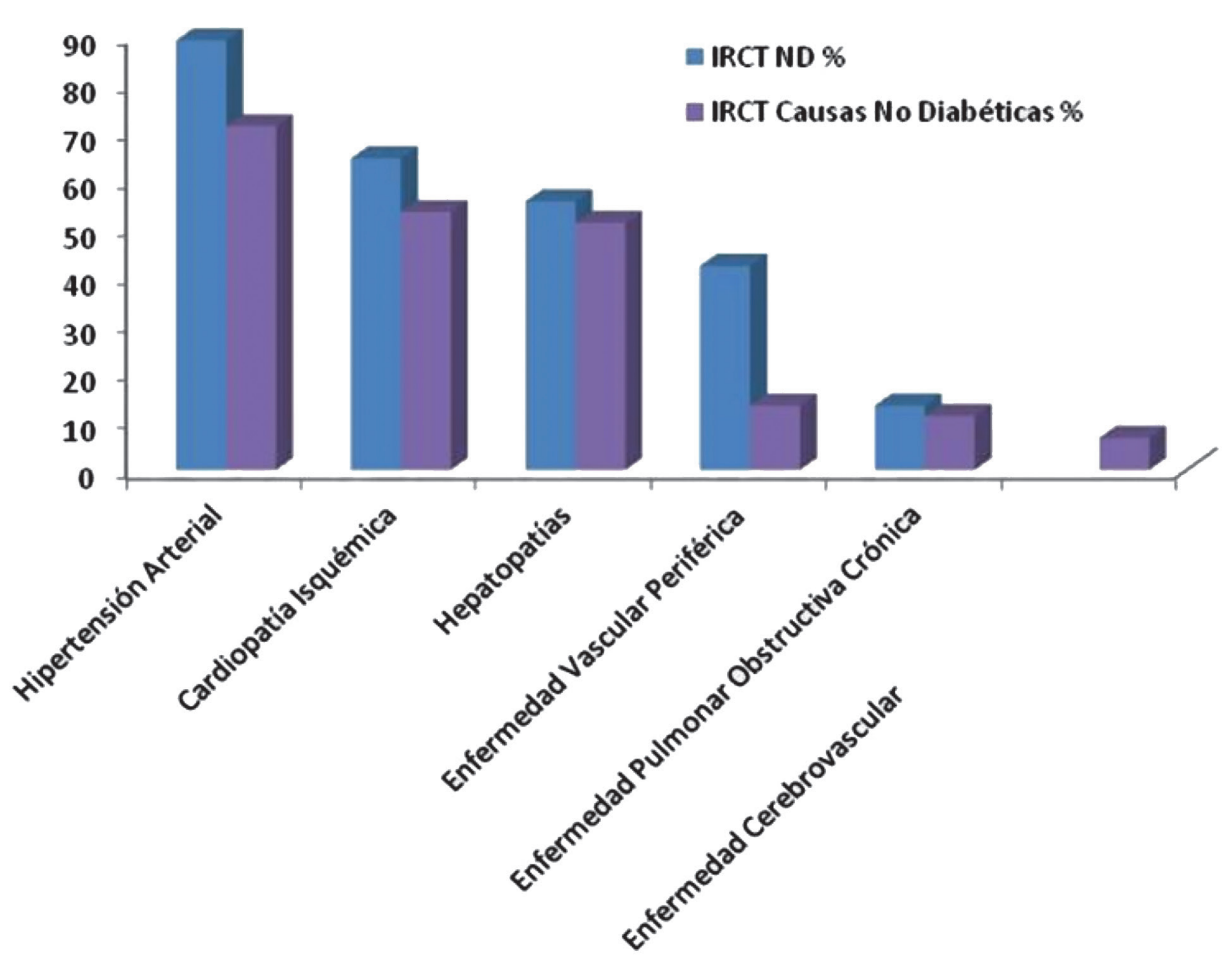

Figura 2. Porcentajes de comorbilidades en los pacientes con nefropatía diabética y causas no diabéticas.

La disfunción del sistema nervioso autónomo y la disfunción diastólica cardíaca del paciente diabético provocan mayor número de hipotensiones en hemodiálisis que en diálisis peritoneal. Estos episodios de hipotensión pueden tener efectos muy negativos en los pacientes diabéticos y provocar cuadros de angina, o isquemia intestinal, cerebral o de miembros inferiores. Al mismo tiempo, estas hipotensiones hacen que se acorte el tiempo de diálisis y, por lo tanto, que el paciente quede "infradializado" y que acumule más líquido y más toxinas urémicas ${ }^{59}$.

La presencia de vasculopatía periférica afecta entre el 20 y el $40 \%$ de los pacientes diabéticos en diálisis. Su presencia causa gran morbimortalidad, ya que, en muchas ocasiones, se asocia a cardiopatía isquémica y puede precisar de múltiples intervenciones, como bypass, angioplastia o amputación de miembros inferiores. Un estudio publicado recientemente, realizado con 29.838 pacientes en diálisis, concluyó que había una gran prevalencia $(6 \%$ ) y una altaincidencia $(2$ eventos $/ 100$ pacientes-año en riesgo) de amputaciones de miembros inferiores entre los pacientes en diálisis y que este riesgo se multiplicaba por nueve en los pacientes diabéticos ${ }^{60-62}$. Los factores clásicos que se asociaron a las amputaciones fueron la edad, la enfermedad vascular periférica y el tabaco. Como factores específicos de la diálisis se asoció el mayor tiempo en diálisis y la alteración del metabolismo óseo y mineral. En los pacientes diabéticos, ser varón, fumador y tener otras complicaciones crónicas de la diabetes, así como la presencia de anemia o malnutrición, se asociaron a un mayor riesgo de sufrir amputaciones de miembros inferiores ${ }^{60,61}$.

Se pueden adoptar medidas para evitar la hipotensión intradiálisis en los pacientes diabéticos 
y así mejorar la tolerancia a dicho proceder. Estas son, entre otras, valorar cuidadosamente el peso seco (peso que debe tener el paciente al acabar la diálisis, una vez que se elimina el exceso de líquido del cuerpo, con buena tolerancia por parte del paciente y sin producir hipotensión), motivar al paciente para que no tenga excesivas ganancias de peso interdiálisis, evitar las ultrafiltraciones agresivas, aumentar el número de sesiones o su duración, evitar la toma de fármacos hipotensores antes de la diálisis, mantener un adecuado control de la anemia y de la nutrición y usar perfiles de sodio en el baño de diálisis que permitan manejar mejor el volumen ${ }^{62-64}$.

En la figura 3, con relación a los pacientes estudiados según Estado Nutricional, se observó un mayor número de casos con sobrepeso ligero $(11,12 \%)$ en la población diabética (IRCT con ND), seguido por pacientes con bajo peso (10\% de esta población). Son notables algunos casos de obesidad.
Muchos estudios han demostrado que los pacientes diabéticos en diálisis presentan mayor prevalencia de malnutrición, especialmente los pacientes diabéticos tipo 2 , con pérdida de proteína somática (reflejada en un descenso de la creatinina plasmática) y de proteína visceral (detectada por un descenso en la albúmina y la prealbúmina). Por tanto, pese a las restricciones propias de la diálisis y la diabetes, hay que proporcionar a estos pacientes un aporte calórico adecuado, con dietas que eviten su malnutrición: $25-30 \mathrm{kcal} / \mathrm{kg} /$ día, $50 \%$ carbohidratos complejos. El contenido proteico será de 1,3-1,5 g/kg/día. Por otro lado, la gastroparesia diabética puede provocar una absorción errática de los alimentos, produciendo episodios tanto de hiperglucemia como de hipoglucemia. Por ello, para mejorar los síntomas, se recomienda a estos pacientes ingerir los alimentos en pequeñas cantidades y varias veces al día ${ }^{63-65}$.

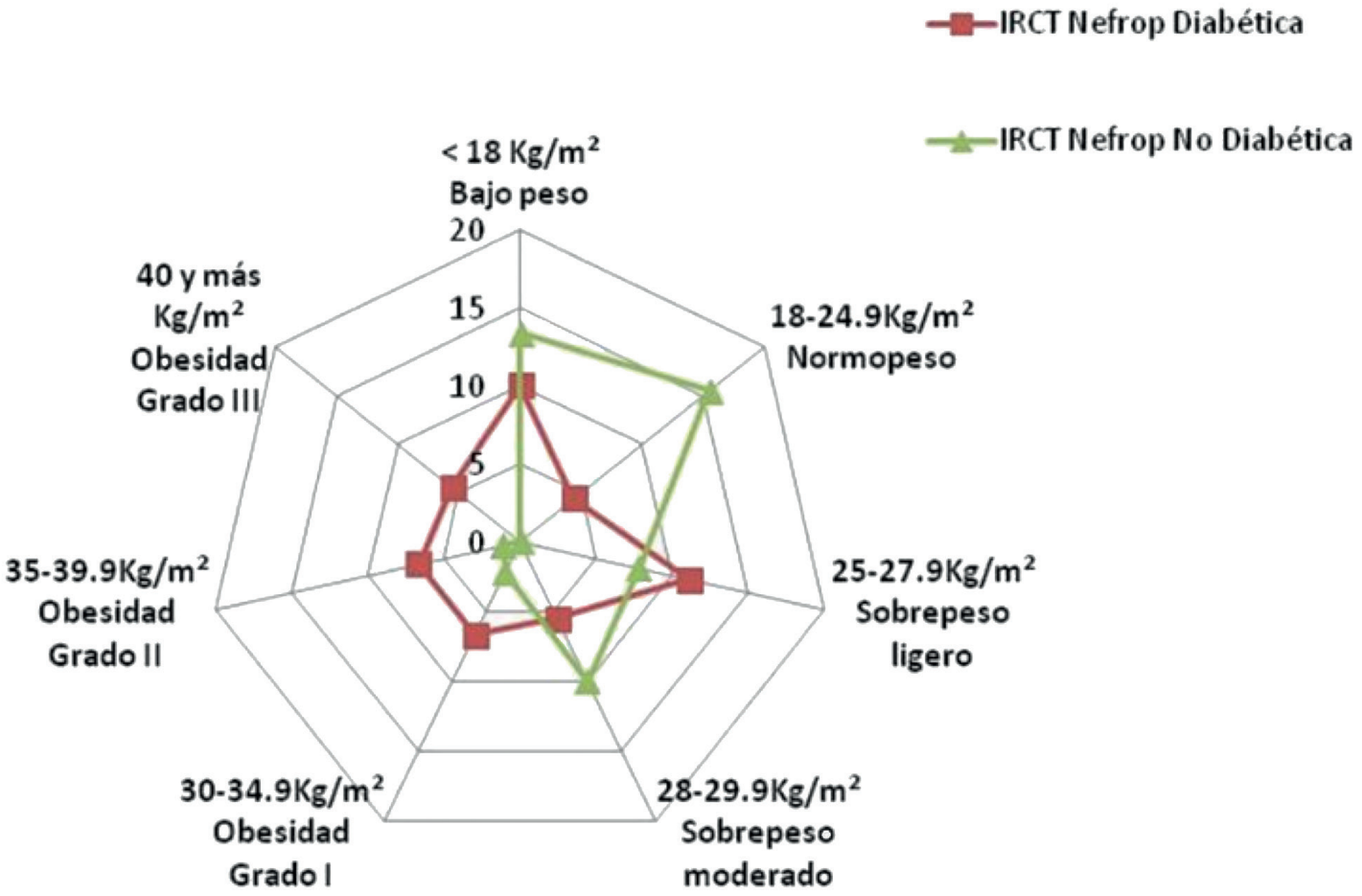

Figura 3. Relación de los pacientes estudiados según Estado Nutricional. 
La figura 4a se refiere a la distribución de los pacientes estudiados según tipo de AV. Se muestra una mayor cantidad de pacientes diabéticos con catéter intravascular de doble luz (17,76\%), en relación con el predominio de los pacientes con fístula arteriovenosa en los no diabéticos $(20,00$
\%). Con relación a la distribución de los pacientes según el número de intentos de $\mathrm{AV}$ permanente (figura 4b), refleja que el $24,45 \%$ de los pacientes diabéticos tuvo entre seis y diez intentos y el 17,76 $\%$, más de 10 intentos. Estas cifras son superiores a las de los no diabéticos.

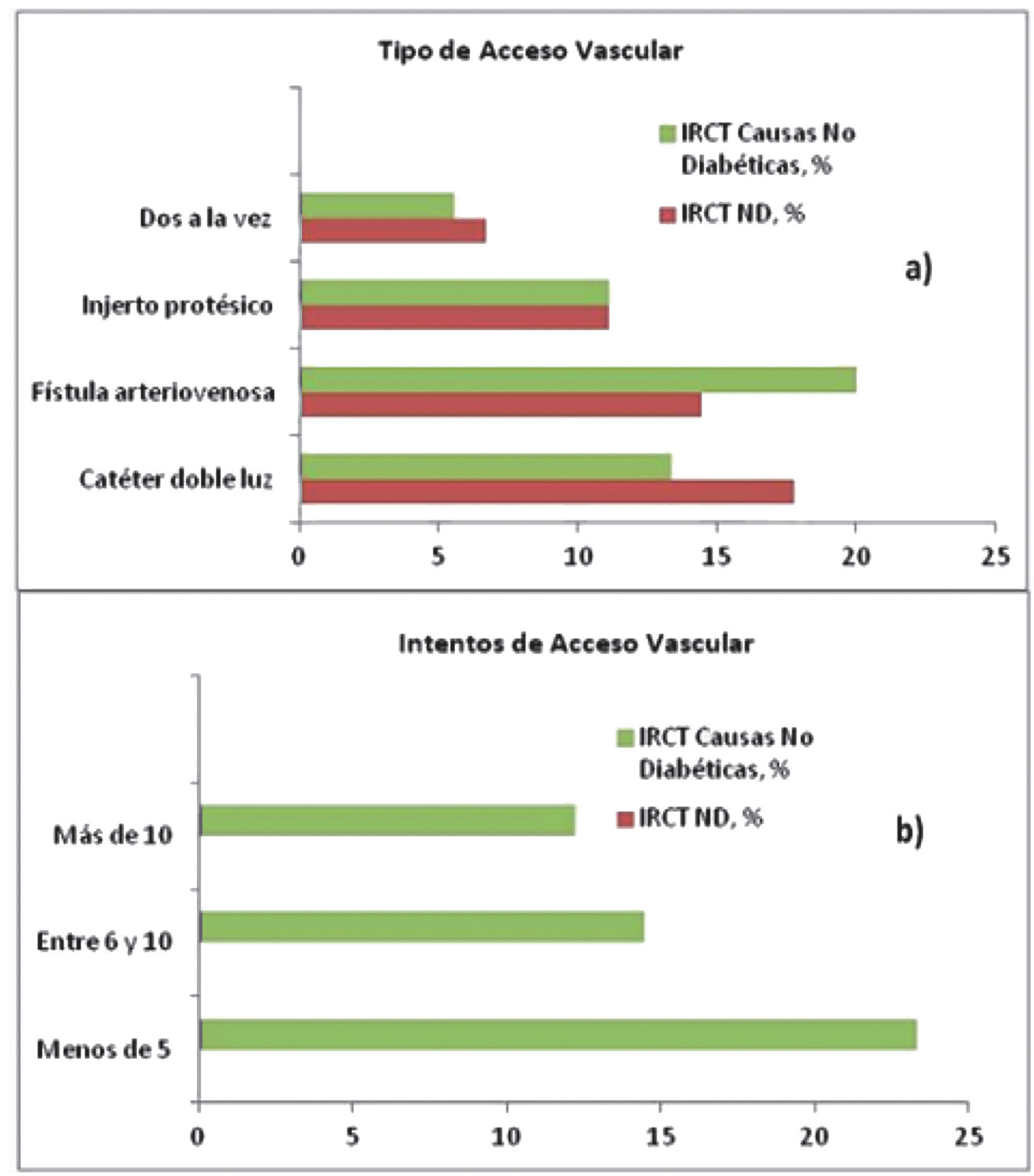

Figura 4. Relación de pacientes estudiados según a) tipo de acceso vascular y

b) intentos de acceso vascular. 
La realización del AV para hemodiálisis supone, en muchas ocasiones, un problema. Los pacientes diabéticos presentan una mayor incidencia de arteriopatía, con una mayor calcificación de las arterias distales de la muñeca (radiocefálica) y con mejor preservación de las arterias proximales (humerocefálica) ${ }^{65}$. Además, tienen un peor sistema venoso, favorecido en parte por la gran cantidad de extracciones sanguíneas y tratamientos intravenosos que han tenido que soportar. Numerosas publicaciones destacan que los pacientes diabéticos presentan un mayor índice de complicaciones en las fístulas arteriovenosas (FAV), del tipo disfunción, trombosis o robo (que consiste en aparición de dolor en la mano, con frialdad y, en ocasiones, lividez, que empeora durante la diálisis, debido al insuficiente riego arterial hacia la zona distal). Estas circunstancias obligan al nefrólogo y al cirujano vascular a plantearse cuál sería el mejor acceso vascular para hemodiálisis en estos pacientes. En general, se recomienda que, en los pacientes en los que no exista contraindicación, tras una valoración somera, se realice, en primer lugar, una FAV radiocefälica, teniendo presente su menor duración. Como segunda alternativa, se plantea la realización de una FAV humerocefálica. Como última opción, porque presenta más complicaciones, se contempla la creación de una fistula protésica o la colocación de un catéter venoso central ${ }^{63-65}$.

En muchos pacientes, el AV resulta complicado debido al insuficiente flujo arterial, como consecuencia de la arterioesclerosis y del mal estado venoso que presentan muchos pacientes con DM. Este problema es mucho más acusado en pacientes que no consultaron de manera oportuna al nefrólogo, dado que ese retraso impide una adecuada planificación del acceso vascular e impone la necesidad de colocar catéteres temporales que pueden comprometer el futuro de los AV permanentes. La supervivencia de injertos vasculares sistémicos, en los pacientes diabéticos, es inferior a la de los pacientes no diabéticos ${ }^{65}$.

En relación con los pacientes estudiados, según complicaciones asociadas a la HD (figura 5), pudo verse que predominaron en la población diabética la hipotensión, la hipoglucemia y las infecciones ( $88,00 \%, 78,00 \%$ y $71,11 \%$, respectivamente). En los no diabéticos, la hipotensión y las infecciones se presentaron con mayor frecuencia.

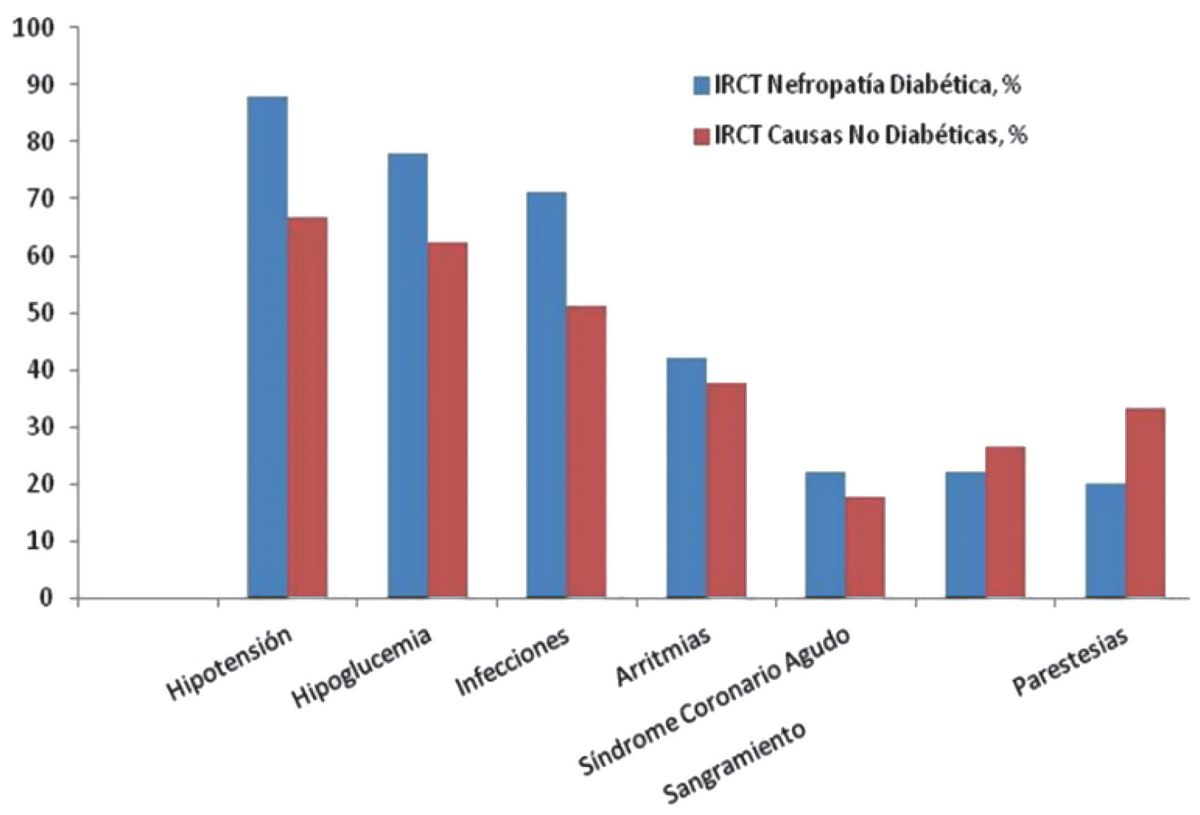

Figura 5. Relación de pacientes estudiados según complicaciones asociadas a la hemodiálisis. 
Entre las principales causas de hipotensión, en los pacientes diabéticos que están sometidos a hemodiálisis, tenemos la peor adaptación a la disminución de volumen plasmático de la diálisis, debido a la disfunción autonómica, la presencia de anemia, la hipoalbuminemia por malnutrición, o por un síndrome nefrótico asociado. Este hecho disminuye la presión oncótica, retarda el rellenado vascular y dificulta la recuperación de la hipotensión ${ }^{64,65}$.

Con respecto al control de la glucemia en estos pacientes, las recomendaciones actuales de la Asociación Americana de Diabetes indican mantener un control metabólico con hemoglobinas glucosiladas (HbAlc) por debajo del $7 \%$, con el fin de reducir las complicaciones microvasculares. Pero se aceptan HbAlc por encima del $7 \%$ en pacientes con historia de hipoglucemias graves, una menor expectativa de vida, comorbilidades asociadas y complicaciones microvasculares $\mathrm{o}$ macrovasculares importantes, como podría ser la ND evolucionada. Los estudios disponibles al respecto, en pacientes diabéticos en diálisis, ofrecen resultados contradictorios. Mientras algunos muestran una peor supervivencia asociada a un peor control glucémico, otros no encuentran correlación en este sentido. Por otro lado, hay varios trabajos que señalan que el estado urémico de estos pacientes puede, por sí mismo, aumentar la formación de HbA1c y que el uso de eritropoyetina puede afectar también a la eritropoyesis y a la formación de HbA1c. Por todo ello, recientemente, se ha sugerido el uso de un factor de corrección para calcular la HbA1c, con base en las dosis de eritropoyetina y en el estado de la anemia. A pesar de estas limitaciones, la HbA1c sigue considerándose el mejor índice para el control de la diabetes, incluso en pacientes con enfermedad crónica terminal ${ }^{63}$.

Existe un nuevo concepto llamado "diabetes quemada" o burntout diabetes. Consiste en una alteración de la homeostasis de la glucemia, que se produce en pacientes con enfermedad renal terminal. Este fenómeno se traduce en la disminución de las necesidades de insulina, con hipoglucemias espontáneas, en pacientes con diabetes avanzada. Hay varios factores que podrían explicar estas alteraciones: el descenso del FG, que reduce el aclaramiento de insulina y aumenta su vida media; la reducción del aclaramiento hepático de insulina por las toxinas urémicas; la disminución de la gluconeogénesis renal; la pérdida de peso y de masa muscular y las restricciones dietéticas, entre otros. Aunque no está claramente demostrado el significado práctico del síndrome de diabetes quemada, algunos autores opinan que los pacientes que lo padecen tienen peor pronóstico y mayor mortalidad que aquellos diabéticos que permanecen hiperglucémicos en diálisis ${ }^{64}$.

En cuanto al tratamiento de la diabetes en pacientes en diálisis, los requerimientos de insulina disminuyen a medida que se deteriora la función renal, por lo que su ajuste debe hacerse de forma progresiva e individualizada para cada paciente. La mayoría de los antidiabéticos orales se eliminan por vía urinaria, por lo que están contraindicados cuando la función renal disminuye. No hay estudios de estos fármacos en pacientes en diálisis $\mathrm{y}$, aunque la repaglinida se puede utilizar en los casos con FG muy reducidos, tampoco hay estudios en pacientes en diálisis. Algunos ensayos con un pequeño número de pacientes han mostrado que los inhibidores de la dipeptidilpeptidasa IV, como vildagliptina, pueden ser utilizados en pacientes en diálisis, aunque en dosis menor que en la población normal ${ }^{65}$.

Por último, respecto a la distribución de los pacientes según principales causas de invalidez para el TR, se pudo ver cómo, en ambos grupos, fue mayor la cantidad de pacientes no aptos para injerto renal por enfermedad cardiovascular, edades extremas e infección activa. Se encontró un mayor número de diabéticos con enfermedades cardiovasculares e infecciones $(66,67 \%$ y $48,89 \%$, respectivamente). 
En pacientes con DM e IRCT, las opciones de trasplante son de riñón y de páncreas, o bien de riñón aislado. La elección de un trasplante u otro dependerá de la edad y del tipo de diabetes del paciente, aunque siempre será esencial realizar un estudio minucioso para determinar las ventajas y desventajas de cada modalidad de trasplante para el receptor. El TR aislado comporta una notable mejoría de la calidad de vida del receptor, pero no influye sobre el control metabólico, ni impide las complicaciones crónicas asociadas a la diabetes. El trasplante simultáneo de páncreas y riñón es un procedimiento más complejo, con mayor riesgo quirúrgico y mayor morbilidad en el periodo posttrasplante. Sin embargo, conlleva algunos beneficios: se alcanza la independencia de la insulina, se evita la recidiva de la nefropatía diabética sobre el riñón trasplantado, se consigue estabilizar o mejorar las complicaciones secundarias y se mejora el control de los factores de riesgo cardiovascular, con la consiguiente disminución de la morbimortalidad a largo plazo $^{63-65}$.

Inicialmente, sólo eran aceptados para TRpáncreas los pacientes con una edad inferior a 45 años. Más tarde, con la mejora de los resultados, este límite se amplió a los 50 años, que, en general, se sigue manteniendo actualmente. En los pacientes de mayor edad se aconseja el trasplante de riñón aislado. No obstante, es bien sabido que no siempre coincide la edad cronológica con la edad biológica, ya que un paciente joven puede haber desarrollado complicaciones más graves que otro de mayor edad, que condicionan un peor pronóstico. Así pues, en ocasiones, el TR-páncreas puede estar contraindicado en un paciente joven, pero indicado en otro de mayor edad, lo que hace imprescindible la valoración individualizada ${ }^{65}$.

Otros parámetros que deben valorarse antes de indicar el tipo de trasplante son: el estado de las complicaciones diabéticas y la presencia y la gravedad de estas complicaciones en el momento en que el paciente es estudiado para trasplante.
La vasculopatía severa es la complicación que más puede condicionar la decisión. En ocasiones, las contraindicaciones para el TR-páncreas o TR aislado pueden ser las mismas desde el punto de vista de la enfermedad cardiovascular.

La implantación de dos órganos requiere una cirugía más compleja y un tiempo de anestesia más prolongado e implica una mayor probabilidad de presentar algún tipo de complicación, o necesitar una reintervención quirúrgica. Por otra parte, la presencia de severas calcificaciones en los vasos iliacos, donde se realizan habitualmente las anastomosis vasculares de los órganos, así como la existencia de una vasculopatía periférica grave, pueden permitir, desde el punto de vista técnico, la implantación de un injerto, pero hacer desaconsejable la implantación de los dos. En estos casos, siempre se da prioridad al TR.

\section{Conclusiones}

Se observó que, en el caso de los pacientes con insuficiencia renal crónica terminal por nefropatía diabética, predominaron los mayores de 60 años, de sexo masculino y con una permanencia de seis meses a un año en el plan de hemodiálisis. La comorbilidad encontrada mayormente fueron la hipertensión arterial, la cardiopatía isquémica y las hepatopatías, en ese orden, en ambos grupos.

Los pacientes renales crónicos por nefropatía diabética estaban distribuidos entre infrapeso y sobrepeso ligero, cerca de la mitad, mientras que un tercio de los no diabéticos reportaron normopeso. El número de pacientes con catéter doble luz y con, al menos, seis intentos de acceso vascular prevaleció en la población diabética estudiada. En los no diabéticos predominaron la fístula arteriovenosa y menos intentos de acceso vascular.

Las complicaciones que con mayor frecuencia se presentaron en el caso de los renales crónicos por nefropatía diabética fueron: hipotensión 
arterial, hipoglucemia e infecciones. En los no diabéticos fueron predominantes, con un porciento significativamente menor, hipotensión e infecciones. La enfermedad cardiovascular y las edades extremas de los pacientes invalidaron, en mayor medida, el trasplante renal en ambos grupos.

\section{Financiación}

El trabajo no tuvo ninguna financiación.

\section{Conflicto de intereses}

Los autores declaran no tener conflicto de intereses en la realización de este artículo.

\section{Responsabilidades éticas}

\section{Protección de personas y animales}

Los autores declaran que para esta investigación no se han realizado experimentos en seres humanos ni en animales.

\section{Confidencialidad de los datos}

Los autores declaran que en este artículo no aparecen datos de pacientes.

\section{Derecho a la privacidad y consentimien- to informado \\ Los autores declaran que en este artículo no aparecen datos de pacientes.}

\section{Contribución de los autores}

Milene Benítez Méndez: control y evaluación de pacientes diabéticos y no diabéticos en control de hemodiálisis.

Dashiell Millet Torres: control y evaluación de pacientes diabéticos y no diabéticos en control de hemodiálisis.

Leonardo Curbelo Rodríguez: control y evaluación de pacientes diabéticos y no diabéticos en control de hemodiálisis.

Francisco Prieto García: redacción y conformación del artículo y estilo y adecuación. 


\section{Referencias}

1. Girach A, Manner D, et al. Diabetic microvascular complications: can patients risk be identified? A review. Int J Clin Pract. 2010;60(11):1471-83. Disponible en: https://doi.org/10.1111/j.1742-1241.2006.01175.x

2. Villar E, Chang SH, et al. Incidences, treatments, outcomes, and sex effect on survival in patients with end-stage renal disease by diabetes status in Australia and New Zealand (1991 2005). Diabetes Care. 2007;30(12):3070-6. Disponible en: https://doi. org/10.2337/dc07-0895

3. Katherine Carranza, Dolores Veron, Alicia Cercado, Noemi Bautista, Wilson Pozo, Alda Tufro, Delma Veron. Aspectos celulares y moleculares de la nefropatía diabética, rol del VEGF-A. Nefrología. 2015;35(2):131-8. Disponible en: https://doi.org/10.1016/j. nefro.2015.05.013

4. Sochett EB, Cherney DZ, et al. Impact of renin angiotensin system modulation on the hyperfiltration state in type 1 diabetes. J Am Soc Nephrol. 2006;17(6):1703-9. Disponible en: https://doi.org/10.1681/ASN.2005080872

5. Pérez-García R, Martín-Malo A, Fort J, Cuevas X, Lladós F. Baseline characteristics of an incident haemodialysis population in Spain: results from ANSWER a multicenter, prospective, observational cohort study. Nephrol Dial Transplant. 2009;24:578-88. Disponible en: https://doi.org/10.1093/ndt/gfn464

6. Correa Rotter R. Nefropatía diabética. En: Treviño Becerra A. Tratado de nefrología. México, DF: El Prado. 2003. t1:663.

7. Pérez-García R, Martín-Malo A, Fort J, Cuevas X, Lladós F. Baseline characteristics of an incident haemodialysis population in Spain: results from ANSWER - a multicentre, prospective, observational cohort study. Nephrol Dial Transplant. 2009;24:570-5.

8. Carranza K., Verón D., Cercado A., Bautista N., Pozo W., Trufo A., Verñon D. M. Aspectos celulares y moleculares de la nefropatía diabética, rol del VEGF-A. Nefrología. 2015;35(2):131-8. Disponible en: https://doi.org/10.1016/j.nefro.2015.05.013

9. Hossain P, Kawar B, El Nahas M. Obesity and diabetes in the developing world. A growing challenge. N Engl J Med. 2007;356:2135. Disponible en: https://doi.org/10.1056/NEJMp068177

10. Tong Z, Yang Z, Patel S Et Al. Promoter polymorphism of the erytropoietin gene in severe diabetic eye and kidney complications. Proc Natl Acad Sci USA. 2008;105:6998-7003. Disponible en: https://doi.org/10.1073/pnas.0800454105

11. Sociedad Chilena de Nefrología. Registro de diálisis. XXV. Cuenta de Hemodiálisis Crónica en Chile. Agosto, 2005.

12. Rodríguez Constantín A, Rodríguez Beyrís RP. Insuficiencia renal crónica en pacientes con diabetes mellitus de tipo 2 en un área de salud.MEDISAN. 2009;13(6).

13. Gu D, Reynolds K, Duan X, Xin X, Chen J, Wu X, et al. Prevalence of diabetes and impaired fasting glucose in the Chinese adult population: International Collaborative Study of Cardiovascular Disease in Asia (InterASIA). Diabetologia. 2008 Sep [citado 15 Jun 2009];46(9):1190-8.

14. Domínguez E, Seuc A, Aldana D, Licea M, Díaz O, López L. Impacto de la diabetes sobre la duración y calidad de vida de la población cubana: años 1990, 1995, 2000 y 2003. Rev Cubana Endocrinol [serie en Internet]. 2006 Ago [citado 17 Jun 2009];17(2).

15. World health report 2003. Chapter 6: Neglected global epidemics: three growing threats. Geneva: WHO; 2003.

16. Seuc A, Domínguez E, Díaz O. Esperanza de vida ajustada por diabetes. Rev Cubana Endocrinol. 2003;14:32-6.

17. Díaz Díaz O. Importancia de los estudios de mortalidad en diabéticos. Rev Cubana Endocrinol. 2001;12:137-8.

18. World health report 2003. Chapter 6: Neglected global epidemics: three growing threats. Geneva: WHO; 2003.

19. Brenner BM, Cooper ME, De Zeew D et al, for the RENAAL Study Group. Effects of losartan on renal and cardiovascular outcomes in patients with type 2 diabetes and nephropathy. N Engl J Med. 2011;345(12):861-9. Disponible en: https://doi.org/10.1056/ NEJMoa011161

20. Effects of ramipril on cardiovascular and microvascular outcomes in people with diabetes: results of the HOPE study and MICRO-HOPE substudy. Lancet. 2010;355, 253-9.

21. Wantier JL, Guillausseau RJ. Advanced glycation end products, their receptors and diabeticangiopathy. Diabetes Metab. 2008;29:86-7.

184 Comportamiento del paciente diabético en hemodiálisis, hospital provincial de Camagüey, Cuba 
22. Kalousova M, Zima T, Tesar V, Dusilova-Sulkova S, Skrha J. Advanced glycoxidation end products in chronic diseases-clinical chemistry and genetic background. Mutat Res. 2009;579:37-46. Disponible en: https://doi.org/10.1016/j.mrfmmm.2005.03.024

23. Setter SM. Biochemical Pathways for microvascular complications of diabetes mellitus. Ann Pharmacother. 2010;37:1858-66. Disponible en: https://doi.org/10.1345/aph.1D002

24. Zerbini G, Bonfanti R, et al. Persistent renal hypertrophy and faster decline of glomerular filtration rate precede the development of microalbuminuria in type 1 diabetes. Diabetes. 2007;55(9):2620-5. Disponible en: https://doi.org/10.2337/db06-0592

25. Ewens KG, George RA, et al. Assessment of 115 candidate genes for diabetic nephropathy by transmission/ disequilibrium test. Diabetes. 2005;54(11):3305-18. Disponible en: https://doi.org/10.2337/diabetes.54.11.3305

26. Gerth J, Cohen CD, et al. Collagen type VIII expression in human diabetic nephropathy. Eur J Clin Invest. 2007;37(10):767-73. Disponible en: https://doi.org/10.1111/j.1365-2362.2007.01864.x

27. Ichinose K, Kawasaki E, et al. Recent advancement of understanding pathogenesis of type 1 diabetes and potential relevance to diabetic nephropathy. Am J Nephrol. 2007;27(6):554-64. Disponible en: https://doi.org/10.1159/000107758

28. Remón C, Quirós PL, Portolés R. Consecuencias. y factores relacionados con la remisión tardía en la enfermedad renal crónica. Nefrología. 2009;29(S5):57-61.

29. Tzamaloukas AH, Friedman EA. Manual de diálisis. In: Daugirdas JT, Blake PG, eds. Diabetes, 2nd ed. Barcelona: Masson, 2003; $472-85$.

30. Hemodialysis Adequacy 2006 Work Group. Clinical Practice Guidelines for Hemodialysis Adequacy. Am J Kidney Dis. 2006;48:390 .

31. Tzamaloukas AH, Friedman EA. Manual de diálisis. In: Daugirdas JT, Blake PG, eds. Diabetes, 2nd ed. Barcelona: Masson, 2013; 472-85.

32. Lassalle M, Labeeuw M, Frimat L, Villar E, Joyeux V, Couchoud C, et al. Age and comorbidity may explain the paradoxical association of an early dialysis start with poor survival. Kidney Int. 2010;77:700-7. Disponible en: https://doi.org/10.1038/ki.2010.14

33. Martínez-Castelao A, Górriz JL, García-López F. Perceived health-related quality of life and comorbidity in diabetic patients starting dialysis (CALVIDIA study). J Nephrol. 2014;17:1-8.

34. Depner TA. Refinements and application of urea modeling. In: Depner TA, ed. Prescribing hemodialysis: a guide to urea modelling. Boston: Kluwer, 2011; 167-94.

35. Konner K. Primary vascular access in diabetic patients: an audit. Nephrol Dial Transplant. 2010;15:1317-25. Disponible en: https://doi.org/10.1093/ndt/15.9.1317

36. Díaz-López M, Plaza-Martínez A, Riera-Vázquez R, Juliá-Montoya J, Manuel-Rimbau E, Corominas-Roura C, et al. Utilidad de las fístulas arteriovenosas autólogas para hemodiálisis en pacientes diabéticos. Angiología. 2002;54:301-7. Disponible en: https:// doi.org/10.1016/S0003-3170(02)74756-1

37. Leehey DJ. Hemodialysis in the diabetic patient with end-stage renal disease. Renal Fail. 2014;16:547-53. Disponible en: https:// doi.org/10.3109/08860229409044884

38. Standards of Medical Care in Diabetes 2010. Diabetes Care. 2010;33:4-10. Disponible en: https://doi.org/10.2337/dc10-S004

39. Drechsler C, Krane V, Ritz E, März W, Wanner C. Glycemic control and cardiovascular events in diabetic hemodialysis patients. Circulation. 2009;120:2421-8. Disponible en: https://doi.org/10.1161/CIRCULATIONAHA.109.857268

40. Lubowsky ND, Siegel R, Pittas AG. Management of glycemia in patients with diabetes mellitus and CKD. Am J Kidney Dis. 2007;50:865-79. Disponible en: https://doi.org/10.1053/j.ajkd.2007.08.012

41. Joy MS, Cefalu WT, Hogan SL, Nachman PH. Long-term glycemic control measurements in diabetic patients receiving hemodialysis. Am J Kidney Dis. 2012;39:297-307. Disponible en: https://doi.org/10.1053/ajkd.2002.30549

42. Kovesdy CP, Park JC, Kalantar-Zadeh K. Glycemic control and burnt-out diabetes in ESRD. Semin Dialysis. 2010;23:148-56. Disponible en: https://doi.org/10.1111/j.1525-139X.2010.00701.x

43. Lou LM, Gota R, Álvarez R, Gutiérrez Colon JA. Parámetros nutricionales de los pacientes en diabéticos en hemodiálisis. Nefrología. 2010;15:385-6. 
44. Watanabe Y, Yuzawa Y, Mizumoto D, Tamai H, Itoh Y, Kumon S, et al. Long-term followup study of 268 diabetic patients undergoing haemodialysis, with special attention to visual acuity and heterogeneity. Nephrol Dial Transplant. 2013;8:725-34. Disponible en: https://doi.org/10.1093/ndt/8.8.725

45. Jaar BG, Astor BC, Berns JS, Powe NL. Predictors of amputation and survival following lower extremity revascularization in hemodialysis patients. Kidney Int. 2004;65:613-20. Disponible en: https://doi.org/10.1111/j.1523-1755.2004.00420.x

46. Combe C, Albert JM, Bragg-Gresham JL, Andreucci VE, Disney A, Fukuhara S, et al. The burden of amputation among hemodialysis patients in the Dialysis Outcomes and Practice Patterns Study (DOPPS). Am J Kidney Dis. 2009;54:680-92. Disponible en: https://doi.org/10.1053/j.ajkd.2009.04.035

47. Bernal Pedreño E, Salces Sáez E, Sambruno Giráldez A. Exploración del pie a los pacientes diabéticos en una unidad de hemodiálisis. Rev Soc Esp Enferm Nefrol. 2009;12:111-6.

48. Jones CA, Krolewski AS, Rogus J, Xue JL, Collins A, Warram JH. Epidemic of end-stage renal disease in people with diabetes in the United States population: do we know the cause? Kidney Int. 2010;67:1684-91. Disponible en: https://doi.org/10.1111/j.15231755.2005.00265.x

49. US Renal Data System. USRDS 2008 Annual Data Report. National Institutes of Health, National Institute. of Diabetes and Digestive and Kidney Diseases, Bethesda, MD, 2008. Disponible en: www.usrds.org

50. Gaede P, Vedel P, Larsen N, Jensen GV, Parving HH, Pedersen O. Multifactorial intervention and cardiovascular disease in patients with type 2 diabetes. N Engl J Med. 2013;30:383-93.

51. Levin A. Consequences of late referral on patient's outcomes. Nephrol Dial Transplant. 2010;15(Suppl 3):8-13. Disponible en: https://doi.org/10.1093/oxfordjournals.ndt.a027977

52. Foley RN, Murray AM, Li S, Herzog CA, McBean AM, Eggers PW, et al. Chronic kidney disease and the risk for cardiovascular disease, renal replacement, and death in the United States Medicare population, 1998 to 1999. J Am Soc Nephrol. 2005;16:489-95. Disponible en: https://doi.org/10.1681/ASN.2004030203

53. Meyer KB, Levey AS. Controlling the epidemic of cardiovascular disease in chronic renal disease: report from the National Kidney Foundation Task Force on Cardiovascular Disease. J Am Soc Nephrol. 2008;9(Suppl):S31-42.

54. Foley RN, Wang C, Collins AJ. Cardiovascular risk factor profi les and kidney function stage in the US general population: the NHANES III study. Mayo Clin Proc. 2005;80(10):1270-7. Disponible en: https://doi.org/10.4065/80.10.1270

55. US Renal Data System. USRDS 2006 Annual Data Report. Atlas of End Stage Renal Disease in the United States, National Institutes of Health, National Institute of Diabetes and Digestive and Kidney Diseases, Bethesda, MD, 2006. Am J Kidney Dis. 2006;47(Suppl 1):S1.

56. Locatelli F, Pozzoni P, Del Vecchio L. Renal replacement therapy in patients with diabetes and end-stage renal disease. J Am Soc Nephrol. 2014;15(Suppl 1):S25-9. Disponible en: https://doi.org/10.1097/01.ASN.0000093239.32602.04

57. Kimmel Pl, Varela MP, Rolf A, Peterson Rl, Weihs KL, Simmens SJ, et al. Interdialytic weight gain and survival in hemodialysis patients: effects of duration of ESRD and diabetes mellitus. Kidney Int. 2010;57:1141-51. Disponible en: https://doi.org/10.1046/ j.1523-1755.2000.00941.x

58. Seliger SL, Weiss NS, Gillen DL, Kestenbaum B, Ball A, Sherrard DJ. HMGCoA reductase inhibitors are associated with reduced mortality in ESRD patients. Kidney Int. 2012;61:297-304. Disponible en: https://doi.org/10.1046/j.1523-1755.2002.00109.x

59. Mason NA, Bailie GR, Satayathum S, Bragg-Gresham JL, Akiba T, Akizawa T, et al. HMG-coenzyme A reductase inhibitor use is associated with mortality reduction in hemodialysis patients. Am J Kidney Dis. 2010;45:119-26. Disponible en: https://doi. org/10.1053/j.ajkd.2004.09.025

60. Wanner C, Krane V, März W, for the German Diabetes and Dialysis Study Group. Atorvastatin in patients with type 2 diabetes mellitus undergoing hemodialysis. N Engl J Med. 2010;353:238-48. Disponible en: https://doi.org/10.1056/NEJMoa043545

61. Jaar BG, Coresh J, Plantinga LC. Comparing the risk for death with peritoneal dialysis and hemodialysis in a national cohort of patients with chronic kidney disease. Ann Intern Med. 2010;143:174. Disponible en: https://doi.org/10.7326/0003-4819-143-3200508020-00003

186 Comportamiento del paciente diabético en hemodiálisis, hospital provincial de Camagüey, Cuba 
62. Sutherland DER, Gruessner RWG, Dunn DL, Matas AJ, Humar A, Kandaswamy R, et al. Lessons learned from more than 1000 pancreas transplants at a single institution. Ann Surg. 2011;233:463-501. Disponible en: https://doi.org/10.1097/00000658200104000-00003

63. Odorico JS, Sollinger HW. Technical and Immunosuppressive advances in transplantation for insulin-dependent diabetes mellitus. World J Surg. 2012;26:194-211. Disponible en: https://doi.org/10.1007/s00268-001-0207-0

64. American Diabetes Association. Pancreas transplantation in type 1 diabetes. Diabetes Care. 2014;27(Suppl 1):105.

65. Fernández-Balcells M, Esmatjes E, Ricart MJ, Casmitjamna R, Astudillo E. Fernández-Cruz L. Successful pancreas and kidney transplantation: a view of metabolic control. Clin Transplant. 2008;12:582-7. 